\title{
Surprising Results of the EMPA-REG OUTCOME study have brought a New Insight into Use of Sodium-Glucose Co-transporter 2 Inhibitors in Patients with Type 2 Diabetes
}

Yuji Aoki*

Department of Internal Medicine, National Hospital Organization Matsumoto Medical Center Matsumoto Hospital, Matsumoto, Nagano, Japan

*Corresponding author: Yuji Aoki, Department of Internal Medicine, National Hospital Organization Matsumoto Medical Center Matsumoto Hospital, 2-2-30 Minami, Murai, Matsumoto City, Nagano Prefecture 399-8701, Japan, Tel: +81 026358 4567; Fax: +81 026386 3183; E-mail: yaoki55@nifty.com

Received date: October 16, 2015, Accepted date: October 23, 2015, Published date: October 26, 2015

Copyright: (c) 2015 Aoki. This is an open-access article distributed under the terms of the Creative Commons Attribution License, which permits unrestricted use, distribution and reproduction in any medium, provided the original author and source are credited.

\begin{abstract}
A surprising new study EMPA-REG OUTCOME has shown that empagliflozin, one of sodium-glucose cotransporter 2 (SGLT2) inhibitors, in addition to standard care had beneficial effects on cardiovascular morbidity and mortality in patients with type 2 diabetes at high risk for cardiovascular events. Subgroup analyses of the study revealed a better hazard ratio for the primary outcome (death from cardiovascular causes, nonfatal myocardial infarction, or nonfatal stroke) in Asian than Caucasian and in patients with age $\geq 65$ years, body-mass index $<30$, glycated hemoglobin $<8.5 \%$ or higher cardiovascular risk than with the respective counterparts. Patients on diuretics also had the hazard ratio favoring empagliflozin, similar to that of patients not on diuretics. I infer that in poorlycontrolled diabetic patients, pre-existing osmotic diuresis is supposed to be augmented by the administration of SGLT2 inhibitors, possibly leading to an acceleration of their dehydration in spite of amelioration of hyperglycemia. Hypovolemia is inferred to be more likely to occur due to osmotic diuresis without an increase in blood glucose level to retain water. It may be recommended that use of SGLT2 inhibitors should be avoided in diabetic patients with glycated hemoglobin $\geq 8.5 \%$ at high risk for cardiovascular events. Because empagliflozin seems to have had no significant effect on nonfatal myocardial infarction or stroke, its ability to reduce cardiovascular mortality may be mediated through osmotic diuresis. SGLT2 inhibitors could be used as a new oral osmotic diuretic to excrete water with a little sodium into urine for non-diabetic patients with heart failure as well. Such action of SGLT2 inhibitors seems to be rather close to that of tolvaptan to promote water diuresis. Thus, the EMPA-REG OUTCOME study has brought a new insight into use of SGLT2 inhibitors in patients with type 2 diabetes.
\end{abstract}

Keywords: EMPA-REG Outcome; Sodium-glucose co-transporter 2 inhibitor; Osmotic diuresis; Cardiovascular mortality; Type 2 diabetes

\section{Commentary}

Surprising results of the EMPA-REG OUTCOME study have been published in the New England Journal of Medicine on September 17, 2015, at NEJM.org [1]. The study (median observation time, 3.1 years) has shown that empagliflozin, one of sodium-glucose co-transporter 2 (SGLT2) inhibitors, in addition to standard care had beneficial effects on cardiovascular morbidity and mortality in a total of 7020 patients with type 2 diabetes (glycated hemoglobin level, 7.0 to $10.0 \%$ ) at high risk for cardiovascular events. The empagliflozin group has demonstrated a significant reduction in the rates of death from cardiovascular causes (38\% relative risk reduction), hospitalization for heart failure (35\% relative risk reduction), and death from any cause (32\% relative reduction). Recent similar trials with other glucoselowering drugs have demonstrated non-inferiority for cardiovascular outcomes, including the TECOS trial with sitagliptin [2], a dipeptidyl peptide-4 inhibitor, and the ELIXA trial with lixisenatide [3], a glucagon-like peptide 1 receptor agonist.

In Japan, however, unexpected adverse effects of SGLT2 inhibitors were reported frequently soon after the first one was marketed in April 2014. Dehydration, which seemed to be occasionally linked to fatal adverse events, was added as a severe adverse effect to a package insert in January 2015. In a position statement of "Management of hyperglycemia in type 2 diabetes, 2015" [4], it is noted that SGLT2 inhibitors with a diuretic effect should be used cautiously in the elderly, in any patient already on a diuretic, and in anyone with a tenuous intravascular volume status. It is surprising that subgroup analyses of the EMPA-REG OUTCOME study [1] revealed a better hazard ratio for the primary outcome (death from cardiovascular causes, nonfatal myocardial infarction, or nonfatal stroke) in Asian than Caucasian and in patients with age $\geq 65$ years, body mass index $<30$, or higher cardiovascular risk than with the respective counterparts. Patients on diuretics also had the hazard ratio favoring empagliflozin, similar to that of patients not on diuretics.

SGLT2 inhibitors decrease hyperglycemia independently of insulin by increasing urinary glucose excretion through the inhibition of glucose reabsorption in the proximal renal tubule. Familial renal glucosuria with normoglycemia is often referred to as a natural model for SGLT2 inhibition. Plasma volume depletion resulting from osmotic diuresis was indicated by activation of the renin-angiotensinaldosterone system in cases of severe renal glucosuria (>10 g/1.73 $\mathrm{m}^{2} / 24 \mathrm{~h}$ ) with a favorable prognosis $[5,6]$. Such activation has been shown after the administration of empagliflozin in type 1 diabetic patients [7]. I infer that in poorly-controlled diabetic patients, preexisting osmotic diuresis is supposed to be augmented by the administration of SGLT2 inhibitors, possibly leading to an acceleration of their dehydration in spite of amelioration of hyperglycemia. Hypovolemia is inferred to be more likely to occur due to osmotic diuresis without an increase in blood glucose level to retain water [8]. 
Citation: Aoki Y (2015) Surprising Results of the EMPA-REG OUTCOME study have brought a New Insight into Use of Sodium-Glucose Cotransporter 2 Inhibitors in Patients with Type 2 Diabetes. Trop Med Surg 3: 199. doi:10.4172/2329-9088.1000199

Page 2 of 2

In subgroup analyses of the EMPA-REG OUTCOME study, it is of note that the primary cardiovascular outcomes were increased in patients with glycated hemoglobin $\geq 8.5 \%$ who received empagliflozin [1]. It may be recommended that use of SGLT2 inhibitors should be avoided in diabetic patients with glycated hemoglobin $\geq 8.5 \%$ at high risk for cardiovascular events. It seems to be a plausible option for such patients to decrease carbohydrate intake by 50 to $100 \mathrm{~g}$ of carbohydrate per day during hyperglycemia, instead of excreting a similar amount of glucose into urine with SGLT2 inhibitors [8], especially for Asian people who preferentially eat carbohydrate-rich foods.

Then, how was empagliflozin working for the secondary prevention of cardiovascular events in the EMPA-REG OUTCOME study? SGLT2 inhibitors have some advantages including modest weight loss, low risk of hypoglycemia and mild decrease of blood pressure [9]. However, in the study, all patients received standard care by optimal blood-pressure lowering and glucose-lowering therapy with agents other than SGLT2 inhibitors. Several mechanisms behind the cardiovascular benefits of empagliflozin were raised in the discussion of the original article [1], such as changes in arterial stiffness and cardiorenal function. Because empagliflozin did not seem to have any effect on nonfatal myocardial infarction and stroke, some clinical researchers think that its beneficial effect on cardiovascular mortality was mediated through osmotic diuresis probably as a class effect of all SGLT2 inhibitors [10]. I agree with this idea, and expect that SGLT2 inhibitors can be used as a new oral osmotic diuretic to excrete water with a little sodium into urine [11-13] for non-diabetic patients with heart failure as well. Such diuretic action of SGLT2 inhibitors, possibly hard to cause electrolyte imbalance, seems to be rather close to that of tolvaptan, a selective oral vasopressin $\mathrm{V}_{2}$-receptor antagonist to promote water diuresis or aquaresis (excretion of electrolyte-free water) [14]. Tolvaptan was effectively and safely added to sodium diuretics for a short period in patients with decompensated heart failure $[15,16]$. Thus, the EMPAREG OUTCOME study has brought a new insight into use of SGLT2 inhibitors in patients with type 2 diabetes. Still caution and prudence should be required for its adverse effects such as genital infection increased in the study.

Conflict of Interest: The author declares that he has no conflict of interest.

\section{References}

1. Zinman B, Wanner C, Lachin JM, Fitchett D, Bluhmki E, et al. (2015) Empagliflozin, Cardiovascular Outcomes, and Mortality in Type 2 Diabetes. N Engl J Med. Sep 17 [Epub ahead of print].

2. Green JB, Bethel MA, Armstrong PW, Buse JB, Engel SS, et al. (2015) Effect of Sitagliptin on Cardiovascular Outcomes in Type 2 Diabetes. N Engl J Med 373: 232-242.
3. Jacob A (2015) The ELIXA trial: lixisenatide safe for cardiovascular disease. http://www.hcplive.com/conference-coverage/esc-2015/the-elixatrial-lixisenatide-safe-for-cardiovascular-disease

4. Inzucchi SE, Bergenstal RM, Buse JB, Diamant M, Ferrannini E, et al. (2015) Management of hyperglycemia in type 2 diabetes, 2015: a patientcentered approach. Update to a position statement of the American Diabetes Association and the European Association for the Study of Diabetes. Diabetes Care 38: 140-149.

5. Scholl-Bürgi S, Santer R, Ehrich JH (2004) Long-term outcome of renal glucosuria type 0: the original patient and his natural history. Nephrol Dial Transplant 19: 2394-2396.

6. Calado J, Sznajer Y, Metzger D, Rita A, Honan MC, et al. (2008) Twentyone additional cases of familial renal glucosuria: absence of genetic heterogeneity, high prevalence of private mutations and further evidence of volume depletion. Nephrol Dial Transplant 23: 3874-3879.

7. Cherney DZ, Perkins BA, Soleymanlou N, Maione M, Lai V, et al. (2014) Renal hemodynamic effect of sodium-glucose cotransporter 2 inhibition in patients with type 1 diabetes mellitus. Circulation 129: 587-597.

8. Aoki Y (2015) Administration of sodium-glucose co-transporter 2 inhibitors could accelerate dehydration in poorly-controlled diabetic patients, proposing an option not to increase glucosuria but to decrease carbohydrate intake during hyperglycemia. Diabetes Res Open J 1: 72-74.

9. Mikhail N (2014) Place of sodium-glucose co-transporter type 2 inhibitors for treatment of type 2 diabetes. World J Diabetes 5: 854-859.

10. Nainggolan L (2015) After EMPA-REG: docs dissect diabetes study, discuss implications. http://www.medscape.com/viewarticle/851453

11. BERNSTEIN LM, BLUMBERG B, ARKIN MC (1958) Osmotic diuretic treatment of refractory edema. Circulation 17: 1013-1020.

12. List JF, Woo V, Morales E, Tang W, Fiedorek FT (2009) Sodium-glucose cotransport inhibition with dapagliflozin in type 2 diabetes. Diabetes Care 32: 650-657.

13. Lin B, Koibuchi N, Hasegawa Y, Sueta D, Toyama K, et al. (2014) Glycemic control with empagliflozin, a novel selective SGLT2 inhibitor, ameliorates cardiovascular injury and cognitive dysfunction in obese and type 2 diabetic mice. Cardiovasc Diabetol 13: 148.

14. Schrier RW, Gross P, Gheorghiade M, Berl T, Verbalis JG, et al. (2006) Tolvaptan, a selective oral vasopressin $\mathrm{V}_{2}$-receptor antagonist, for hyponatremia. N Engl J Med 355: 2099-2112.

15. Matsuzaki M, Hori M, Izumi T, Fukunami M (2011) Efficacy and safety of tolvaptan in heart failure patients with volume overload despite the standard treatment with conventional diuretics: a phase III, randomized, double-blind, placebo-controlled study (QUEST study). Cardiovasc Drugs Ther Suppl 1: S33-S45.

16. Patra S, Kumar B, Harlalka KK, Jain A, Bhanuprakash HM, et al. (2014) Short term efficacy and safety of low dose tolvaptan in patients with acute decompensated heart failure with hyponatremia: a prospective observational pilot study from a single center in South India. Heart Views 15: $1-5$. 